\title{
INFLUÊNCIA DO TREINAMENTO DE FLEXIBILIDADE E FORÇA MUSCULAR EM ATLETAS DE GINÁSTICA RÍTMICA
}

\section{Jessica Miranda Silva}

Graduada em Educação física Faculdade Metropolitana de Maringá (FAMMA), Maringá (PR), Brasil.

\section{Daniel Vicentini de Oliveira}

Doutorado em Gerontologia (UNICAMP); Docente no Departamento de Educação Física da Faculdade Metropolitana de Maringá (FAMMA).

E-mail: d.vicentini@hotmail.com

\section{Daniel Eduardo Cunha Leme}

Mestrado em Gerontologia (UNICAMP).

José Roberto Andrade do Nascimento Júnior

Doutor em Educação física (UEM); Docente no Departamento de Educação física da Universidade Federal do Vale do São Francisco (UNIVASF).

\section{Ana Luiza Barbosa Anversa}

Doutorado em andamento em Educação física (UEM); Docente no Departamento de Educação física da Faculdade Metropolitana de Maringá (FAMMA).
RESUMO: O estudo teve por objetivo analisar a influência de um treinamento na flexibilidade e força muscular de atletas de ginástica rítmica (GR). Trata-se de uma pesquisa quase experimental, composta por 15 atletas de GR, de 09 a 13 anos. Para avaliação da flexibilidade foi utilizado o Teste de Sentar e Alcançar e o Teste de Flexibilidade Adimensional. Para avaliação da força muscular, foi utilizado o teste de impulsão vertical e horizontal. Foi realizada avaliação pré e pósintervenção, com os testes citados acima. O protocolo de intervenção foi composto de exercícios específicos de flexibilidade e força muscular. Verificou-se que tanto a flexibilidade linear $(p=0,009)$ quanto à flexibilidade adimensional $(p=0,001)$ melhoraram após a intervenção. Houve diferença significativa também na impulsão horizontal $(p=$ $0,001)$ e vertical $(p=0,001)$. Por meio dos resultados adquiridos, pode-se concluir que o programa de exercícios de intervenção se refletiu de forma positiva na flexibilidade e a força muscular das atletas de GR.

PALAVRAS-CHAVE: Educação física; Esporte; Exercício físico.

\section{THE INFLUENCE OF TRAINING ON MUSCULAR FLEXIBILITY AND FORCE IN RHY THM GYMNASTIC ATHLETES}

\begin{abstract}
Current study analyzes the influence of training on muscular flexibility and force in athletes of rhythm gymnastic (RG). The experimental research comprised 15 RG athletes between 9 and 13 years old. The Sitting and Reaching Test and A-dimensional Flexibility Test were employed to assess flexibility. Vertical and horizontal impulse test was used to assess muscular force. Pre- and post-intervention were undertaken for the above-mentioned tests. Intervention protocol consisted of specific exercises in flexibility and muscular force. Linear $(p=0.009)$ and a-dimensional $(p=0.001)$ flexibility improved after intervention. There was also a significant difference in horizontal $(p=0.001)$ and vertical $(p=0.001)$ impulse. Results showed that intervention exercises were positive on flexibility and muscular force of RG athletes.
\end{abstract}

KEY WORDS: Physical exercise; Sports; Physical education.

\section{INTRODUÇÃO}

A ginástica rítmica é um esporte que requer altos níveis de coordenação motora, resistência muscular, agilidade, flexibilidade, 
força, equilíbrio e impulsão para execução dos movimentos técnicos e artísticos da modalide (DOS SANTOS et al., 2016).

A flexibilidade em específico é necessária para o sucesso na modalidade e para a perfeita realização dos gestos acrobáticos (BLUM; BEAUDOIN, 2000). Nos últimos ciclos olímpicos nota-se que o nível técnico da GR tem evoluído (LANARO FILHO; BÖHME, 2001), sendo de suma importância pesquisas e ações direcionadas a este âmbito. Toledo e Antualpa (2016) apontam que o ciclo olímpico (2013-2016) tem dado maior atenção a aspectos técnicos e sequência de elementos do que questões coreográficas e artísticas, o que remete à importância da flexibilidade e força para atender a demanda coreográfica da modalidade.

$\mathrm{Na}$ GR, as ginastas bucam o rendimento e a performance física máxima, por estes motivos há treinamentos de habilidades específicas. Ademais, há uma busca pelo aprimoramento estético e artístico dos movimentos, por meio dos exercícios individuais e de conjunto (DE SOUZA MENEZES; NOVAES; FERNANDESFILHO, 2012).

Além da flexibilidade, a força muscular contribui para a execução de movimentos eficientes e manutenção do equilíbrio corporal, além de correlacionar positivamente com a qualidade de vida dos praticantes. A literatura ressalta que a flexibilidade e a força muscular são qualidades físicas importantes para a promoção da saúde e também para o bom desempenho no esporte competitivo (CARVALHO et al., 1998).

A melhora dessas capacidades físicas auxilia no treinamento promovendo maior facilidade na execução técnica dos movimentos. Para De Souza Hirata e Oliveira (2015) o treinamento direcionado para a Ginástica Rítmica baseia-se nos princípios da biomecânica articular, tendo em vista aumentar a amplitude do movimento, propiciando ganho de força, flexibilidade e resistência muscular.

Para a melhora da força muscular, os exercícios resistidos estão entre os mais indicados. Os mesmos são praticados e desenvolvidos mundialmente. Tal fato pode ser explicado pelos vários benefícios decorrentes de sua prática, que incluem modificações morfológicas, neuromusculares, fisiológicas, alterações sociais e comportamentais (SILVA et al., 2015).

Em estudo realizado por Simões et al. (2016), sobre o estado da arte das pesquisas em ginástica, constata-se que os artigos se direcionam para a pedagogia do esporte, formação e atuação profissional, aspectos fisiológicos e nutricionais. Entretanto, nota-se que mesmo com as publicações direcionadas para fisiologia e influência das capacidades físicas, poucos estudos debatem a influência de um programa de treinamento na flexibilidade e força na ginástica rítmica. Diante disso, o estudo teve como objetivo analisar a influência de um treinamento na flexibilidade e força muscular de atletas de ginástica rítmica.

\section{METODOLOGIA}

Trata-se de uma pesquisa do tipo quantitativa, quase experimental, aprovada pelo Comitê de Ética em Pesquisa do Centro Universitário Cesumar (UniCesumar) por meio do parecer 878.856/2014.

A amostra deste estudo, escolhida por conveniência, foi composta por 15 atletas do sexo feminino da seleção maringaense de ginástica rítmica, com idade entre 09 e 13 anos, sendo cinco da categoria pré-infantil (treinam 12 horas/semana), cinco da categoria infantil (treinam 12 horas/semana) e cinco da categoria juvenil (treinam 16 horas/semana). Foram selecionadas para compor a amostra as ginastas que apresentaram melhor desempenho e resultados significantes nos campeonatos importantes da modalidade na maioria das competições, por exemplo, campeonatos regionais. A inclusão se deu a partir de um convite verbal feito diretamente aos responsáveis pelos treinamentos e pela aprovação dos pais à participação das ginastas na coleta de dados, mediante assinatura do Termo de Consentimento Livre e Esclarecido (TCLE).

Para avaliação da flexibilidade foi utilizado o Teste de Sentar e Alcançar, que possui o propósito de medir o grau de flexibilidade com flexão à região anterior do quadril com ambos os joelhos extendidos, sem flexionar durante o movimento (GUEDES, 2006). Para tal feito, foi utilizada uma caixa de madeira com dimensões de 30 $\mathrm{cm} \times 30 \mathrm{~cm}$, tendo a parte superior uma tábua fixa de 
$56 \mathrm{~cm}$ de comprimento, sobre a qual se fixa uma escala de medida de até $50 \mathrm{~cm}$, sendo que o avaliado deverá acomodar-se com as mãos para iniciar o teste no valor 23 que condiz com a linha onde o avaliado posicionará os pés (DELGADO, 2004).

Para a realização do teste, o avaliado deveria estar descalço e sentado de frente para o banco, com os joelhos estendidos debaixo da caixa e com os pés totalmente em contato com o banco. O avaliador estabilizava os joelhos do avaliado na tentativa de segurar que estes não flexionariam durante o movimento de extensão. Os braços foram estendidos sobre a superfície da caixa com as mãos posicionadas uma sobre a outra, palma da mão voltada para baixo e os dedos posicionados na mesma altura. Para que fosse registrado o resultado, o avaliado, em contato com a caixa, estendia-se para frente ao longo da escala de medida, procurando alcançar o seu limite, maior distância possível sem tirar a mão da fita, deslizando e sem solavancos. Foram realizadas três tentativas de flexão de tronco, empurrando a régua com a ponta dos dedos e mantendo os joelhos, cotovelos e punhos em extensão. $O$ avaliado foi orientado a permanecer na distância alcançada por dois segundos, para a leitura da régua (GUEDES, 2006). Não foi considerado o resultado quando o avaliado flexionasse os joelhos durante $o$ movimento de extensão do quadril, ou, quando as mãos não estivessem uma sobre a outra e com as pontas dos dedos na mesma altura. Nestes casos, o teste foi refeito.

Ressata-se que o teste de flexibilidade linear não é suficiente para avaliar a flexibilidade das ginastas, uma vez que este avalia apenas a flexibilidade do quadril e posterior de coxa, sendo assim, optou-se em aprofundar a investigação em relação à flexibilidade utilizando o teste de flexibilidade adimensional (flexiteste), que tem como propósito avaliar a flexibilidade de cada articulação de forma passiva máxima de 20 movimentos. Conforme De Araujo (2000) este teste possui um poder distinto maior do que outros métodos adimensionais, por apresentar um escore igual ou maior que 60 pontos, caracterizandose por apresentar um critério de hipermobilidade generalizada do indivíduo, ou seja, ampla flexibilidade global do corpo quando igual ou maior que 60 pontos.

As medidas adimensionais são um dos protocolos mais frequentemente empregados na monitoração dos níveis de flexibilidade com o propósito de condicionamento físico. Esse protocolo procura oferecer informações sobre os níveis de flexibilidade pela execução de movimentos articulares com alongamento muscular passivo, ou seja, com auxílio de outra pessoa que pode ser o avaliador. São vinte movimentos articulares que abrangem as articulações de tornozelo, joelho, quadris, tronco, punho, cotovelo e ombro, sendo que cada um desses movimentos é retratado por meio de uma escala crescente e descontínua de valores inteiros que varia de 0 a 4 (GUEDES, 2006) $\mathrm{O}$ avaliador conduziu o movimento até o seu ponto máximo de cada articulação. As medidas foram comparadas com as figuras para cada movimento realizado (ROCHA; GUEDES JR, 2013).

A amplitude das medidas realizadas para cada movimento se expressava de acordo com a seguinte escala: 0 - muito baixa; 1 - baixa; 2 - média; 3 - alta; 4 - muito alta. As posições intermediárias entre dois valores quaisquer são medidas pelo menor resultado; um exemplo: se em um movimento cuja amplitude o avaliado ficar entre a posição 2 e 3 , serão medidos como grau 2 (DANTAS, 1999).

O Teste de força explosiva (impulsão vertical) foi utilizado para medir, indiretamente, a força muscular dos membros inferiores. Para a realização do teste de impulsão vertical foi fixada uma fita métrica de três metros em uma parede lisa. $\mathrm{O}$ avaliado se posicionava lateralmente à superfície graduada, com as plantas dos pés totalmente apoiadas sobre o solo, e com um braço completamente estendido acima da cabeça, onde foi marcado o ponto mais alto alcançado com o dedo médio (3 tentativas). Para facilitar a marcação, foi utilizado pó de giz na extremidade dos dedos. A partir da posição ortostática, a execução consistitu em flexionar as pernas e executar a impulsão contra movimento, com auxílio dos braços, e tocar o ponto mais alto possível na parede. $\mathrm{O}$ valor foi calculado pela diferença da maior altura alcançada (com salto) e a altura parado, com os valores expressos em centímetros (GUEDES, 2006).

Já o Teste de força explosiva (impulsão horizontal) foi utilizado para medir a força dos membros inferiores por meio do desempenho em se impulsionar horizontalmente para frente sem corrida de aproximação. Sendo utilizada uma fita métrica no solo e a partir da 
posição inicial anteriormente a fita demarcada com os pés parelelos e afastados à largura dos quadris, o avaliado saltava à frente, com impulso simultâneo das pernas, saltando mais longe possível. A movimentação do tronco e dos braços ficava a critério do avaliado, sem se movimentar após a aterrissagem sobre o solo até que a leitura da medida seja realizada. $\mathrm{O}$ avaliado teve três oportunidades para demarcar o melhor dos três resultados alcançados, entre a linha de partida e a linha do calcanhar que tocou no solo após o salto (GUEDES, 2006).

Após a coleta dos dados no próprio local de treinamento das atletas, foi aplicado um programa de intervenção por meio de exercícios, com duração de oito semanas, duas vezes na semana, 45 minutos de duração por sessão, totalizando 16 sessões, visando o desenvolvimento da força muscular (sete exercícios, direcionados para o trabalho de salto vertical, horizontal, unitaletal, transposição de obstáculos, burpes e atividades no minitrampolim) e exercícios de alongamento, com o objetivo de produzir melhora quanto à flexibilidade (15 exercícios, trabalhados por meio de movimentos técnicos da modalodade como abertura, ponte e equilíbrios, com alongamentos ativos e passivos com auxílio de cordas e elásticos). Após as semanas de intervenção/treinamento foram realizados os pós-testes para verificar e comparar os resultados obtidos.

Os circuitos de intervenção para as habilidades motoras foram desenvolvidos e divididos especificamente para força e flexibilidade. Sendo aplicada a flexibilidade seguida da força com descanço de um circuito para o outro de dois minutos, para que não interferisse no resultado.

O protocolo de flexibilidade foi composto de exercícios de alongamento estático (30 segundos) e dinâmico (12 repetições) de membros superiores em conjunto com inferiores. O circuito de força foi composto de três séries de sete exercícios, tendo duração máxima de 30 segundos para cada exercício, com intervalo de dois minutos entre as séries.

O tratamento dos dados foi realizado a partir dos resultados adquiridos na primeira coleta de testes pré-intervenção, e após o período de dois meses do programa de intervenção, os testes de pós-intervenção.
Para a análise dos dados, foi realizada a análise da distribuição dos dados com base no teste de KolmogorovSmirnov. Como os dados apresentaram distribuição normal, foram utilizados Média (x) e Desvio-padrão (dp) para a caracterização dos resultados. Para comparar o grau de flexibilidade linear e adimensional, e a impulsão vertical e horizontal, foi utilizado o teste de t de student dependente. Para analisar a correlação entre as variáveis, foi utilizado o coeficiente de correlação de Pearson. O nível de significância adotado foi de $p<0,05$.

\section{RESULTADO}

A partir dos dados apresentados na Tabela 1 , verificou-se que tanto a flexibilidade linear $(p=$ $0.009)$ quanto a flexibilidade adimensional $(p=0.001)$ aumentaram após a intervenção, evidenciando que o programa de intervenção motora e de força se mostrou interveniente na melhora da flexibilidade das ginastas.

Também houve diferença significativa na impulsão horizontal $(p=0.001)$ e vertical $(p=0.001)$, indicando que o programa de intervenção influenciou positivamente na impulsão das atletas. Ao analisar a correlação entre as variáveis, não foi encontrada correlação significativa $(p>0,05)$ entre nenhuma das variáveis em ambos os momentos (pré e pós-teste).

Tabela 1. Comparação do grau de flexibilidade e da impulsão horizontal e vertical antes e após o programa de intervenção motora de oito semanas das ginastas da cidade de Maringá (PR)

\begin{tabular}{lccc}
\hline Categoria & $\begin{array}{c}\text { Pré- } \\
\text { intervenção }\end{array}$ & $\begin{array}{c}\text { Pós- } \\
\text { intervenção }\end{array}$ & $\boldsymbol{P}$ \\
\cline { 2 - 3 } & $\mathbf{x} \pm \mathbf{d p}$ & $\mathbf{x} \pm \mathbf{d p}$ & \\
\hline $\begin{array}{l}\text { Flexibilidade } \\
\text { linear (cm) }\end{array}$ & $41,60 \pm 4,26$ & $42,993 \pm 4,18$ & $0.009^{*}$ \\
$\begin{array}{l}\text { Flexibilidade } \\
\text { adimensional } \\
(\mathrm{cm})\end{array}$ & $41,40 \pm 6,58$ & $51,07 \pm 3,43$ & $0.001^{*}$ \\
$\begin{array}{l}\text { Impulsão } \\
\text { horizontal (cm) }\end{array}$ & $144,20 \pm 19,27$ & $172,20 \pm 13,71$ & $0.001^{*}$ \\
$\begin{array}{l}\text { Impulsão vertical } \\
(\mathrm{cm})\end{array}$ & $67,33 \pm 8,64$ & $74,60 \pm 8,67$ & $0.001^{*}$ \\
\hline
\end{tabular}

*Diferença significativa: $p<0,05$ - Teste $\mathrm{t}$ de student dependente. $\mathrm{cm}$ : centímetros; dp: desvio padrão.

Fonte: Dados da pesquisa. 
Ainda com base nos resultados (Tabela 1) notase que as ginastas da amostra estudada apresentaram um alto percentil de flexibilidade quando comparadas aos protocolos utilizados para este estudo, pois praticam regularmente a Ginástica Rítmica, sendo que na flexibilidade linear de todas as 15 atletas foram apresentados resultados acima da média, que, de acordo com Guedes e Guedes (2006), é de 33,5 cm para essa faixa etária, sendo na pré-intervenção média de 41,60 \pm $4,26 \mathrm{~cm}$ e após intervenção, média de 42,93 \pm 4,18.

\section{DISCUSSÃO}

Conforme apresentado nos resultados, a flexibilidade das ginastas apresenta-se acima da média para a faixa etária estudada, uma vez que segundo Karloh et al. (2010), a flexibilidade é uma característica marcante de esportes que necessitam da elasticidade, como a GR. Mesmo encontrando índices acima da média para flexibilidade (linear e adimensional), o programa interventivo aplicado contribuiu para a melhora desta qualidade física assim como para força (impulsão vertical e horizontal) uma vez que foram encontradas direrenças significativas entre o pré e pós-teste. Esses resultados comprovam a eficácia do programa interventivo, uma vez que, além de ser qualidades físicas essenciais para a GR, está presente nos treinos diários e mesmo assim se obteve desenvolvimento físico dessa capacidade física na amostra estudada.

Sabe-se que as atletas de GR são submetidas a um treinamento intenso e repetitivo desde muito jovens (DA SILVA et al., 2009), o que pode determinar consequentes alterações positivas para o esporte, já que os gestos esportivos da modalidade demandam grandes amplitudes articulares, em especial da coluna vertebral (extensão) e articulação coxofemoral (KARLOH et al., 2010).

Estudos realizados por Del Vecchio et al. (2014) e Martins et al. (2009) também encontraram índices de flexibilidade superior em atletas de ginástica rítmica quando comparados a não praticantes da modalidade na mesma faixa etária, ressaltando que o aumento desta capacidade está diretamente relacionada ao tempo de prática e estímulos de treinamento. Os resultados significativos alcançados pela variável flexibilidade adimensional $(p=0.001)$ e linear $(p=0.009)$ foram importantes para o estudo, uma vez que é possível considerar que a intervenção proposta obteve sucesso, mesmo com o perído de invervenção relativamente curto, a melhora obtida vai auxiliar na execução de alguns movimentos, principalmente aqueles exigidos pelo Código de Pontuação durante as coreografias nas competições, além de poder ser utilizada ou dar subsídios para ações interventivas em outros centros de treinamento ou ao longo de um período maior junto à equipe pesquisada.

Weineck (2006) e Herbert (2007) colocam que o treino da flexibilidade é importante para reduzir o risco de lesão e melhorar o desempenho muscular geral, otimizando a fluência dos movimentos e aprendizagem dos gestos técnicos, além disso, os autores destacam que uma flexibilidade mal desenvolvida pode se refletir em uma estagnação do desempenho da atleta.

Quanto à força muscular, os resultados apontaram melhora significativa no teste de impulsão horizontal $(p=$ $0.001)$ e vertical $(p=0.001)$, apresentando a importância do programa interventivo com exercícios de força, além dos realizados comumente nos treinamentos específicos. O desenvolvimento da força muscular em ginastas é necessário para a execução de movimentos adequados da técnica, uma vez que a modalidade exige força explosiva verticalmente e horizontalmente nos saltos, que devem ter grande amplitude dos movimentos, força rápida para execução, força de reação para execução de giros e força isométrica para execução dos equilíbrios. Desta forma, essa capacidade física de força torna-se um dos principais componentes de aptidão física para execução da performance deste esporte. Conforme Santos (2011) a grande maioria dos movimentos da ginástica demanda flexibilidade e intensidade, para que sejam alcançadas de forma adequada é necessário um elevado nível de força, por isso é de suma importância incluir nos treinamentos trabalhos específicos de força, de forma especializada e infividualizada.

De acordo com Astrand (2008), não há uma melhora significativa no treinamento de força em idade pré-púberes, uma vez que com base em Minatto et al. (2010) fatores endógenos interferem na força e 
flexibilidade, diminuindo na puberdade, devido a isso deve-se fundamentar o trabalho em outras capacidades. A diferença na força de atletas pré e pós-puberdade foi encontrada por De Souza Menezes, Novaes e FernandesFilho, ao verificar que atletas não menarcadas apresentam maior força de impulsão vertical do que as menarcadas. Porém não foi o percebido no presente estudo, o que demonstra que o trabalho específico de força pode resultar de forma positiva no treinamento da ginástica rítmica, em especial nos movimentos de saltos que demandam uma boa elevação do corpo, sendo necessário que o impulso realizado sobre o solo seja suficiente para superar a força realizada pela gravidade, para que obtenham grande amplitude de movimento durante o voo (MURAD, 2009).

Perante os resultados encontrados, pode-se apontar que o programa interventivo contribui para o desempenho das atletas nas provas da modalidade, uma vez que os índices de força e flexibilidade apresentaram melhora e podem se refletir na execução de elementos corporais obrigatórios exigidos pelo Código de Pontuação da GR, como ondas, equilíbrios, saltos e pivôs.

Por fim, destaca-se que essa pesquisa se limita por tratar de casos com ginastas de uma única equipe, desta forma, não deve ser considerada representativa de toda a população de ginastas, sendo uma limitação do estudo. Além disso, há a necessidade de futuros estudos que contenham um grupo controle, não praticante de ginástica rítmica. Sugerem-se também estudos semelhantes com outras modalidades esportivas ou com um grupo maior de ginastas de outra região, podendo servir de base para outros esportes que também necessitam das qualidades físicas flexibilidade e força muscular.

\section{CONCLUSÃO}

Por meio dos resultados adquiridos, pode-se concluir que o programa de exercícios de intervenção melhorou a flexibilidade e a força muscular das atletas de Ginástica Rítmica. Por fim, se confirma a hipótese de que o programa interventivo apresenta influência nas qualidades físicas de flexibilidadee força muscular em atletas de Ginástica Rítmica.

\section{REFERÊNCIAS}

ACHOUR JÚNIOR, A. Manual de Instruções-Fleximeteravaliando a flexibilidade. Instituto Code de Pesquisas. Rio de Janeiro: Midiograf, 1997.

ASTRAND, P. O. Crianças e adolescentes: desempenho, mensurações, educação. Rev Bras Ciênc Mov, v. 6, n. 2, p. 59-68, 2008.

BLUM, J. W.; BEAUDOIN, C. M. Does flexibility affect sport injury and performance? Parks \& Recreation, v. 35 , n. 10, 2000.

CARVALHO, A. C. G.; PAULA, K. C.; AZEVEDO, T. M. C.; NÓBREGA, A. C. L. Relação entre flexibilidade e força muscular em adultos jovens de ambos os sexos. Rev Bras Med Esporte, v. 4, n. 1, p. 2-8, 1998.

DOS SANTOS, J. B. et al. Perfil postural de atletas de ginástica rítmica na faixa etária de 10 a 19 anos no estado de São Paulo. RBPFEX, v. 10, n. 59, p. 395-404, 2016.

DANTAS, E. H. Flexibilidade: alongamento e flexionamento. Shape, 1999.

DE ARAÚJO, C. G. S. Correlação entre diferentes métodos lineares e adimensionais de avaliação da mobilidade articular. Rev Bras Ciênc Mov, v. 8, n. 2, p. 25-32, 2000.

DE SOUZA MENEZES, L.; NOVAES, J.; FERNANDESFILHO, J. Qualidades físicas de atletas e praticantes de Ginástica Rítmica pré e pós-púberes. Rev Salud Pública, v. 14, n. 2, p. 238-247, 2012.

DE SOUZA HIRATA, A. C.; DE OLIVEIRA, R. F. Protocolo de treinamento proprioceptivo para atletas de Ginástica Rítmica-GR. ConScientiae Saúde, v. 14, n. 4, p. 634-640, 2015.

DELGADO, L. A. Avaliação da aptidão física: projeto de elaboração de sistema de informação. 2004. 75 f. Monografia (Licenciatura em Educação Física) Departamento de Educação Física, Universidade Federal do Maranhão, São Luís, MA.

GUEDES, D. P. Manual prático para avaliação em 
educação física. [S.l.]: Manole, 2006.

KARLOH, M.; SANTOS, R. P.; KRAESKI, M. H.; MATIAS, T. S.; FRUTUOSO, A. S. Alongamento estático versus conceito Mulligan-efeitos crônicos no treino de flexibilidade em ginastas. Rev Bras Cineantrop Desemp Hum, v. 12, n. 3, p. 202-8, 2010.

LANARO FILHO, P.; BÖHME, M. T. S. Detecção, seleção e promoção de talentos esportivos em ginástica rítmica desportiva: um estudo de revisão. Rev Bras Educ Física e Esporte, v. 15, n. 2, p. 154-168, 2001.

MINATTO, G. et al. Idade, maturação sexual, variáveis antropométricas e composição corporal: influências na flexibilidade. Rev bras cineantropom desempenho hum, v. 12, n. 3, p. 151-8, 2010.

MURAD, V. C. Análise da força explosiva de membros inferiores em atletas de ginática ritimca e ginástica artística feminina. [s.l.; s.n.], 2009.

ROCHA, A.; GUEDES JR, D. Avaliação física para treinamento personalizado, academias e esportes. São Paulo: Phorte, 2013.

SANTOS, A. B. Flexibilidade e força em ginástica rítmica: Avaliação de Ginastas Juniores Portuguesas. 2011.

SILVA, M. B.; LIMA, F. M.; FERNANDES, L. F. R. M.; BERTONCELLO, D. Comparação entre diferentes exercícios resistidos para jovens não treinadas. Saúde e Pesq, v. 8, n. 3, p. 549-556, 2015.

SILVA, L. R. V.; LOPEZ, L. C.; COSTA, M. C. G.; GOMES, Z. C. M.; MATSUSHIGUE, K. A. Avaliação da flexibilidade e análise postural em atletas de ginástica rítmica desportiva flexibilidade e postura na ginástica rítmica. Rev Mackenzie educ fis esporte, v. 7, n. 1, p. 59-68, 2008.

SIMÕES, R. et al. A produção acadêmica sobre ginástica: estado da arte dos artigos científicos. Rev Bras Educ Física e Esporte, v. 30, n. 1, p. 183-198, 2016.

TOLEDO, E.; ANTUALPA, K. The appreciation of artistic aspects of the Code of Points in rhythmic gymnastics: an analysis of the last three decades. Rev Bras Educ Física $\mathrm{e}$ Esporte, v. 30, n. 1, p. 119-131, 2016.

VIEBIG, R. F.; POLPO, A. N.; CORRÊA, P. H. Ginástica Rítmica na infância e adolescência: características e necessidades nutricionais. Lecturas: Educación física y deportes, n. 94, p. 13, 2006.

Recebido em: 01 de julbo de 2016 Versão final recebida em: 08 de agosto de 2016 Aceito em: 16 de agosto de 2016 